\title{
DESAFÍOS CONTEMPORÁNEOS DEL DESARROLLO HUMANO Y SOSTENIBILIDAD: UNA MIRADA DESDE LA BIODIVERSIDAD, AGRICULTURA Y SOBERANÍA ALIMENTARIA.
}

\author{
JUAN PABLO SUAZO ${ }^{1}$ \\ MARVIN MORENO ${ }^{2}$ \\ JOSE DANIEL LARA ${ }^{3}$ \\ PABLO AMILCAR DIAZ ${ }^{4}$
}

Doctorandos en Ciencias del Desarrollo Humano, Universidad Nacional Autónoma de Honduras

DOI: $10.5377 /$ rpdd.v6i1.12440

Recibido: agosto, 2021

Aceptado: septiembre, 2021

\section{RESUMEN}

$\mathrm{E}^{\prime}$ enfoque de desarrollo, desde una perspectiva de acumulación, tiene raíces tempranas a la revolución industrial, pero es posterior a la Segunda Guerra Mundial que se adopta el concepto de desarrollo como sinónimo del crecimiento económico, quedando a merced de una disposición operativa mecanicista relativa al crecimiento. No obstante, el concepto ha evolucionado, de desarrollo humano y, a posteriori desde una perspectiva más integral, a desarrollo humano sostenible, con los que se han adoptado compromisos de los países para alcanzar metas de desarrollo como los Objetivos de Desarrollo del Milenio y los Objetivos de Desarrollo Sostenible 2030, impulsados a nivel global por la Organización de las Naciones Unidas.

1 MSc.JUAN PABLO SUAZO, Docente Universidad Nacional de Agricultura, juanpablosuazo@yahoo.com, Orcid: https://orcid.org/0000-0003-26301928.

2 MSc. MARVIN MORENO, Coordinador Regional para America Latina y el Caribe del Ciclo de Proyectos de la FAO, marvin.morenojimenez@fao.org, Orcid: https://orcid.org/0000-0003-3116-1977.

3 MSc. JOSE DANIEL LARA, Oficial de proyectos DIAKONIA-Honduras, daniel.lara@diakonia.se, Orcid: https://orcid.org/0000-0002-9026-3074

4 MSc. PABLO AMILCAR DIAZ, Docente Universidad Pedagógica Nacional Francisco Morazán, pablodiaz1380@yahoo.com, Orcid: https://orcid.org/0000-0003-1540-9661. 
A través de la historia, la humanidad ha encontrado la forma de utilizar los recursos que le provee el ambiente. La innovación ha sido medio para la sobrevivencia y la modificación de su hábitat, para asegurarse alimentación, salud, seguridad y bienestar. En este proceso, las modificaciones del medio ambiente y el acelerado crecimiento económico y tecnológico han generado tal impacto que se ha dado inicio a la era geológica del Antropoceno. La agricultura forma parte de esas modificaciones, lo que ha permitido satisfacer la seguridad alimentaria, primero con la domesticación de plantas y animales, pero también, por una agricultura tradicional de baja tecnificación fragmentada $y$, por otra parte, una agricultura comercial intensiva que tiene repercusiones sociales económicas y ambientales, que tiene repercusiones sociales económicas y ambientales, lo que ha sido un factor importante para la modificación de los paisajes naturales.

En los últimos cien años en razón del modelo económico hegemónico, sin límites de crecimiento, se ha alterado el estado natural de la ecósfera, de manera tal que se pone en tensión la coexistencia armoniosa, y por ende el equilibrio indispensable, entre la especie humana y la naturaleza.

El presente trabajo hace un análisis de los planteamientos teóricos contemporáneos relacionados con los desafíos del desarrollo humano sostenible en un país biodiverso como Honduras por su parte, pero con procesos preocupantes de deterioro y destrucción de sus ecosistemas, que agregan presión a una situación compleja entre vulnerabilidad social y riesgo ante los desastres naturales.

\section{PALABRAS CLAVE}

Desarrollo Humano, biodiversidad, agricultura, sostenibilidad, soberanía alimentaria

\section{ABSTRACT}

The development approach, from an accumulation perspective, has early roots in the industrial revolution, but it is after the Second World War, that the concept of development is adopted as a synonym for economic growth, being at the mercy of a relative mechanistic operative disposition to growth. However, the concept has evolved from human development and, a posteriori from a more comprehensive perspective, to sustainable human development, with which countries have made commitments to achieve development goals such as the Millennium Development Goals and the Sustainable Development Goals 2030. 
Through history, humanity has found a way to use the resources provided by the environment. Innovation has been a means of survival and modification of their habitat, to ensure food, health, safety and well-being. In this process, environmental modifications and accelerated economic and technological growth have generated such an impact that the geological era of the Anthropocene has begun. Agriculture is part of these modifications, which has made it possible to satisfy food security, first with the domestication of plants and animals, but by a traditional low-tech and fragmented agriculture and, on the other hand, an intensive commercial agriculture. These methods of land uses have economic and environmental-social repercussions and are an important factor in the modification of natural landscapes.

In the last 100 years, due to the hegemonic economic model, without limits to growth, the natural state of the ecosphere has been altered, in such a way that the harmonious coexistence between the human species and nature is put in tension.

This paper makes an analysis of contemporary theoretical approaches related to the challenges of sustainable human development in a biodiverse country like Honduras for its part, but with worrying processes of deterioration and destruction of its ecosystems, which add pressure to a complex situation between social vulnerability and risk of natural disasters.

\section{KEYWORDS}

Human Development, biodiversity, agriculture, sustainability, food sovereignty.

\section{INTRODUCCIÓN}

El Doctorado en Ciencias del Desarrollo Humano (DCDH) de la Universidad Nacional Autónoma de Honduras organizó, en modalidad virtual, el Foro: "Debate Contemporáneo sobre las Ciencias del Desarrollo Humano", el cual se realizó del 10 al 17 de mayo de 2021. En esta actividad académica participaron 14 científicos y docentes del DCDH y de diferentes universidades latinoamericanas y europeas, lo que permitió un abordaje global sobre los principales temas de discusión.

Entre los temas científicos desarrollados se encuentra el debate sobre Biodiversidad, Ambiente y Desarrollo, a través de una jornada de ponencias magistrales sobre los "Retos actuales para la sostenibilidad ambiental y el desarrollo humano desde la perspectiva de la diversidad cultural y biológica, la recuperación 
de sistemas forestales y la soberanía alimentaria". Los especialistas invitados fueron los siguientes: Dra. Sonia Lagos-Witte, Docente del Doctorado en Ciencias del Desarrollo Humano de la Universidad Nacional Autónoma de Honduras, Dra. Olga Lucía Sanabria, Coordinadora del Doctorado en Etnobiología y Estudios Bioculturales de la Universidad del Cauca, Colombia, y Dr. Alejandro Casas, Investigador Titular del Instituto de Investigaciones en Ecosistemas y Sustentabilidad de la Universidad Autónoma de México. Es con base a las exposiciones y discusiones generadas de este foro que se fundamenta el debate teórico e investigativo desarrollado para la construcción del presente artículo.

En el documento se expone un debate teórico y de contexto, a partir de cuatro temas principales y su estructura se presenta en cuatro sub-capítulos: el primero orientado a desarrollo humano y sostenibilidad ambiental, el segundo relacionado a agricultura y sostenibilidad del desarrollo, el tercero aborda la soberanía y seguridad alimentaria de los pueblos desde la perspectiva del desarrollo y en el cuarto capítulo se plantea un análisis de la situación hondureña, respecto a la protección y conservación de la biodiversidad.

\section{Desarrollo humano y sostenibilidad ambiental}

La humanidad, a través de la historia ha innovado y se ha valido de muchas modificaciones para poder satisfacer sus necesidades, enfrentarse a la naturaleza y poder sobrevivir; en los últimos 100 años las dos grandes mediaciones para alcanzar el desarrollo, son el crecimiento económico y el progreso técnico.

Tras la segunda guerra mundial (1940.1945), los organismos multilaterales de crédito y los organismos de cooperación, fomentaron el desarrollo económico y con el apoyo de la Organización de las Naciones Unidas (ONU), colocaron al desarrollo y al crecimiento económico como sinónimos y a la política económica tras el único objetivo: el crecimiento ilimitado de la economía mundial (Rendón, 2007). Como consecuencia de lo anterior, el desarrollo quedó a merced del crecimiento económico como una disposición operativa y mecanicista sin freno. Como lo expresa Guhl (2012), "No existe conciencia sobre los límites asociados a los recursos naturales y estos se utilizan como si fueran infinitos" (p. 382).

Por otra parte, el concepto de desarrollo sostenible se introduce a partir del año 1972, en la conferencia de las Naciones Unidas sobre Medio Ambiente, luego 
se introduce con el informe "La Estrategia Mundial para la Conservación", de 1980; posteriormente este concepto fue popularizado por el informe de la comisión sobre medio ambiente, conocida como La Comisión Brundtland del año 1987. Esta concepción de desarrollo sostenible fue adoptada por la Cumbre para la Tierra de Río de Janeiro, en el año 1992.

La propuesta del desarrollo humano sostenible, planteada hace varias décadas, sin duda representaba un concepto evocador y atractivo, el cual generó muchas expectativas. Sin embargo, esta retórica es buena para la agenda diplomática y para las políticas internacionales, pero en los momentos que se viven actualmente se necesita de acciones más concretas, ante lo que parece inminente, de continuar en esta misma línea de consumismo desmedido, con el riesgo de llegar a un punto de quiebre sin retorno, con relación a la destrucción del medio ambiente y la agudización de los problemas relacionados con el desarrollo humano, como el aumento de la pobreza y la desigualdad social.

El desarrollo sostenible pretende comprender las interacciones entre tres sistemas complejos interactuantes, como lo son: la economía mundial, la sociedad global y el medio ambiente físico de la tierra. La cuestión es ¿cómo encontrar un punto de equilibrio y de convergencia?

En este sentido la agenda 2030 para el Desarrollo Sostenible, elaborada en el 2015 por el Programa de las Naciones Unidas para el Desarrollo (PNUDUNDP), consta de 17 objetivos de desarrollo sostenible (ODS) que abarcan las tres dimensiones de la sostenibilidad, por lo que se convierte en el marco de referencia para el desarrollo sostenible a nivel global (Morazán, 2017). Los ODS presentan, en los objetivos número 11 al número 16, objetivos de carácter ecológico-ambiental. En el contexto actual de la Pandemia por COVID-19 que ha venido a profundizar los problemas, Honduras, como país en vías de desarrollo, enfrenta retos significativos para poder alcanzar los ODS y esos deberán ser revisados y actualizados a la brevedad posible.

Si los recursos naturales, la biodiversidad y todos los diferentes ecosistemas que la naturaleza nos provee, son concebidos como un bien público universal que nos pertenece a todos, es imperativa la obligación de los Estados de asegurar la utilización sostenible de los recursos naturales, con base en la Declaración de Nueva Delhi, acerca de los principios de derecho internacional relativos al desarrollo sostenible del año 2002 (Rodrigo, 2015). Sin embargo, la gigantesca economía mundial está 
provocando una inmensa crisis ambiental, capaz de amenazar la vida y el bienestar de miles de millones de personas, así como la supervivencia de millones de otras especies del planeta, si no la nuestra propia (Vila, 2015).

Desde la revolución industrial, la influencia del hombre en la pérdida de la naturaleza es tan grande que numerosos científicos son de la opinión que estamos en una nueva Era Geológica: el denominado Antropoceno, donde el insaciable apetito por lograr crecer más económicamente, ha alterado significativamente los diferentes ecosistemas del planeta, en detrimento del propio bienestar humano. Estudios recientes demuestran que un $75 \%$ de la superficie no cubierta de hielo ya ha sido alterada, la mayoría de los mares están contaminados y se ha perdido el $85 \%$ de los humedales (WWF, 2020).

La Biodiversidad es un componente de la naturaleza, cuya importancia es incuestionable ya que juega un papel crucial en el aprovisionamiento de alimentos, agua, fibras, materias primas, medicinas, por lo que resulta de fundamental importancia su conservación, así como la preservación de los procesos ecológicos esenciales tales como la polinización, regulación del clima, calidad del agua y el control de la contaminación. Sin Biodiversidad no hay manera de sobrevivir, ni de frenar el Calentamiento Global del planeta. Por lo tanto, debemos hacer conciencia de que el desarrollo humano sostenible depende del equilibrio y la sostenibilidad de la naturaleza, del trabajo que realicemos para reducir los factores que aceleran el cambio climático y de los esfuerzos por frenar el calentamiento global (Lagos-Witte, Sanabria \& Casas, mayo 2021).

El crecimiento económico y el crecimiento sostenible son dos conceptos que parecieran ser inconmensurables después de ver realidades como las de Honduras, donde, si bien es cierto no hay un crecimiento económico considerable que lo posicione como un país desarrollado, presenta un índice de desarrollo humano de 0.634 (PNUD, 2020), que lo ubica en el puesto 132 de 189 países del ranking de Desarrollo Humano. Por otra parte, Honduras es uno de los países más vulnerables a los efectos del cambio climático en la región y del mundo, por lo que se requieren medidas urgentes e integrales para aliviar la crisis que vive la mayoría de la población hondureña.

En palabras de Hodge, Daber, López, Castilla, \& Edwards (2018):

"es fundamental buscar soluciones integrales que consideren las interacciones de los sistemas naturales entre sí y con los sistemas sociales. No hay dos crisis separadas, 
una ambiental y otra social, sino una sola y compleja crisis socioambiental, el desafío urgente de proteger nuestra casa común incluye la preocupación de unir a toda la familia humana en la búsqueda de un desarrollo sostenible e integral” (p.11).

En el Artículo 9 del Acuerdo de Escazú (Organizacion de las Naciones Unidas, 2018) se destaca el derecho de vivir en un medio ambiente sano, un entorno propicio para el trabajo de personas, organizaciones o grupos que promuevan la protección del medio ambiente, proporcionándoles reconocimiento y protección, contribuyendo a la protección de cada persona, de las generaciones presentes y futuras, en un buen medio ambiente y desarrollo sostenible.

El Acuerdo de Escazú se desprende del Tratado de la Convención sobre la Diversidad Biológica que Honduras firmó en la Cumbre para la Tierra de Río de Janeiro de 1992 y posteriormente ratificó. Sin embargo, no ha sido ni firmado, ni ratificado por Honduras, lo cual debilita las acciones para la protección del medio ambiente en el país y requiere una urgente acción por parte del Estado de Honduras. La educación ambiental en los diferentes niveles educativos es de carácter imprescindible para poder generar esos procesos de concientización en las generaciones actuales y futuras sobre la importancia del ambiente. En este sentido Aznar y Barrón (2017) sugieren que, para poder conseguir el compromiso de todos los sectores implicados en la propuesta del Desarrollo Humano Sostenible, es fundamental que la educación y la formación para la sostenibilidad lleguen a todos los sectores y consiga transformar la cultura dominante.

¿Cómo pensar que el desarrollo sostenible es verdaderamente humano, cuando no se tiene el derecho a disfrutar de una naturaleza plena, en óptimas condiciones? La crisis climática que acontece en el planeta tierra es tan peligrosa, como lo es para la vida de las personas y las comunidades que defienden sus territorios naturales. Doscientos doce defensores del medio ambiente fueron asesinados en el año 2019 en Colombia, Brasil, México, Honduras, Guatemala, Venezuela y Nicaragua (Gómez, 2020). Lo anterior convierte a América Latina en la región más peligrosa del mundo para los defensores del ambiente.

\section{Agricultura y sostenibilidad}

La agricultura es por antonomasia la acción antropogénica que ha permitido la evolución de las sociedades desde la prehistoria hasta el postmodernismo. La domesticación de plantas y animales fue el punto de inflexión para el surgimiento 
de las ciudades, siguiendo la Teoría de Childe (1988), citado en Serrano y Stein (2019). El acceso a los alimentos, ya sea por la prodigalidad de la naturaleza o por la domesticación de plantas y animales, dio origen a las migraciones, que a la vez dio forma a las múltiples culturas y a la demografía actual.

El debate sobre el origen de la agricultura se sustenta en varios enfoques teóricos, Olazával (2014) hace una aproximación sobre los debates teóricos acerca del origen de la agricultura que tienen como punto de convergencia la relación entre crecimiento demográfico y agricultura. Lo anterior pone de manifiesto la relación entre agricultura y el ser humano, como expresión antropogénica, a través de la manipulación de los recursos naturales, para la satisfacción de sus necesidades. La agricultura ha sido el vehículo para el desarrollo de la humanidad, sin embargo, la demanda de alimentos y un sistema económico voraz, sin equilibrios entre crecimiento y sustentabilidad, pone en tensión los ecosistemas y la propia sobrevivencia de los seres vivos.

El informe del Club de Roma sobre "Los Límites del Crecimiento" (1972) citado en Iranzo (2005), advierte que el fin del crecimiento económico indiscriminado sucedería en cualquier momento, si no se tomaban las decisiones acertadas, y que por lo tanto para reducir la huella ecológica global, habría que desarrollar una revolución de la sostenibilidad, una manera análoga a la revolución neolítica, que superó el período de caza y recolección, o de la revolución industrial, que lo hizo con la sociedad agraria.

El ser humano ha transformado el paisaje natural a partir de la ampliación de la frontera agrícola para el pastoreo. Después de la Segunda Guerra Mundial, una importante nueva intensificación agrícola, importada de los países desarrollados, comenzó a penetrar en este paisaje del legado histórico (países en vía de desarrollo), complementado por la Revolución Verde (es decir, variedades de alto rendimiento, agroquímicos, y otras tecnologías agrícolas (Hayami \& Ruttan, 1985; Olmstead \& Rhode, 1993; Magdoff \& col.2000, citado en Perfecto \& Vandermeer, 2008).

En términos de equilibrio entre intensificación productiva y biodiversidad, de acuerdo con Perfecto y Vandermeer (2008), se percibe un incremento de la productividad, pero a la vez un decrecimiento de la biodiversidad, especialmente ante la presencia de agricultura industrial, acompañada de tecnologías de la revolución verde (pesticidas, fertilizantes y maquinaria). 


\section{DESAFÍOS CONTEMPORÁNEOS DEL DESARROLLO HUMANO Y SOSTENIBILIDAD: UNA MIRADA DESDE LA BIODIVERSIDAD, AGRICULTURA Y SOBERANÍA ALIMENTARIA.}

La agricultura, como sistema productivo, coexiste en dos mundos paralelos: por un lado, la tecnificación, la digitalización, la biotecnología y otras innovaciones que incrementan exponencialmente la productividad que se concentra en los países ricos, que además la subvencionan, y por otra parte, países pobres que además concentran la mayor cantidad de los agricultores familiares, tienen limitaciones para acceder a estas tecnologías y a las subvenciones, por lo que los enfoques tradicionales de producción basados en la agricultura migratoria y extensiva también ponen en riesgo los ecosistemas.

La agricultura no solo asegura la disponibilidad y el acceso a los alimentos, en el caso de Honduras representa cerca del 13\% del Producto Interno Bruto (Banco Central de Honduras, 2020), principalmente basado en la producción de materias primas o productos básicos como café, banano, aceite de palma, carne de res, azúcar, maíz, leche y otros (Banco Interamericano de Desarrollo, 2019). No obstante, el crecimiento del sector no se fundamenta en la implementación de políticas agroalimentarias que apuestan a la productividad, vía tecnificación, sino en la expansión de la frontera agrícola. La producción de café, aguacate y palma africana en Mesoamérica, soja y carne de res en Brasil, entre otras, viene acompañada de impactos ambientales significativos (Nájar, 2016; Deutsche Welle, 2020).

Alcanzar relaciones homeostáticas entre agricultura y crecimiento económico, demanda de un mayor conocimiento sobre los factores condicionantes de este desequilibrio en los países: i) las brechas en el acceso a tecnologías e innovaciones de agricultores familiares, ii) el impacto ambiental pernicioso, que estos sistemas de explotación generan sobre los ecosistemas, iii) la tensiones sociales generadas a partir de las amenazas a los bienes comunes (agua, bosques, suelo) de las sociedades en su conjunto, y la pérdida de la riqueza biocultural que generan estos sistemas de producción iv) las limitaciones institucionales para la regulación y el cumplimento de las normativas ambientales, v) la falta de compromiso de los países importadores y los propios consumidores, para tener posiciones responsables, de consumo de productos con costos ambientales elevados.

A la par de las brechas tecnológicas, el cambio climático se cierne sobre el futuro de la agricultura, la variabilidad climática (períodos de sequías o inundaciones) afecta negativamente la paupérrima economía familiar y la seguridad alimentaria. Como efecto, las familias abandonan sus tierras y engrosan las corrientes migratorias que se dirigen hacia países con mayores oportunidades o en su defecto a las ciudades, que crecen exponencialmente sin dar espacio a la planificación y a la urbanización. 
A luz de estas realidades, organismos como la FAO promueven la agricultura climáticamente inteligente (CSA, siglas en inglés), que conceptualmente "constituye un enfoque que ayuda a orientar las acciones necesarias para transformar y reorientar los sistemas agrícolas, a fin de apoyar de forma eficaz el desarrollo y garantizar la seguridad alimentaria en el contexto de un clima cambiante". La CSA persigue tres objetivos básicos: el aumento sostenible de la productividad y los ingresos, la adaptación y la creación de resiliencia ante el cambio climático, y la reducciónabsorción de gases de efecto invernadero.

Con la llegada del Cambio Climático, la agricultura debe, por un lado, reducir las emisiones de gases de efecto invernadero que, de acuerdo al Banco Mundial (s.f.) representa entre el $19 \%$ y el $29 \%$ del total de emisiones anuales, y, por otra parte, debe aumentar la resiliencia.

Lagos-Witte et al., (2021) reafirman la preocupación acerca del impacto pernicioso que han tenido las sociedades sobre la transformación de los ecosistemas después de la segunda guerra mundial, lo que obliga a revisar el capitalismo como modelo económico hegemónico que se acompaña del uso indiscriminado de fertilizantes, que genera la eutrofización de las aguas, la sobre explotación de las aguas dulces para riego y la pérdida de especies animales como los insectos. Un cambio de paradigma es necesario, una mirada a la agricultura orgánica o ecológica es importante, considerando que la biodiversidad y la satisfacción de las necesidades humanas son conciliables (Linares-Rosas, Goméz, Adalsoro-Maya, \& Casas, 2021). Por último, tal como lo expresa Al Gore, la era de la revolución de la sostenibilidad ha llegado, la generación de modelos productivos eficientes, equitativos, integrales y respetuosos con el medio ambiente es una necesidad impostergable que alimenta la esperanza de eliminar el hambre, de alcanzar estados de bienestar social, generar riqueza para los países y desde una perspectiva existencialista, asegurar nuestra propia sobrevivencia como especie (CNN, 2021).

\section{Soberanía y la seguridad alimentaria de los pueblos}

El abordaje de la Soberanía Alimentaria (SA) en procesos de desarrollo, plantea amplios desafíos epistemológicos y contextuales que generan debate sobre la evolución de las sociedades y su productividad, a través de sistemas de extensión agrícola, tecnología de los alimentos, uso de sustancias químicas para la producción, trazabilidad temporal y geográfica de los alimentos. Todos estos procesos impactan, de una manera u otra la cosmovisión de los pueblos, la cultura alimentaria, la medicina 
ancestral, las semillas criollas, las prácticas tradicionales de producción de traspatio, la recolección de plantas y frutos, entre otras.

De acuerdo con Guzmán (2008), "La incontenible aceleración de los procesos sociales constituye el cemento con el que se forjan las sociedades capitalistas industriales. Una de las características clave de tales sociedades lo constituye el papel que juega en ellas la innovación científica y tecnológica" (p. 8).

La evolución conceptual sobre soberanía alimentaria, se construye en escuelas de pensamiento de activismo técnico, investigativo y extensión desarrollista, es así que la SA se entiende como:

"el derecho de un país a definir sus propias políticas y estrategias sustentables de producción, distribución y consumo de alimentos, que garanticen el derecho a la alimentación sana y nutritiva para toda la población, respetando sus culturas y la diversidad de los sistemas productivos, de comercialización y lo rural" (FAO, 2013, p.18).

“el derecho de los pueblos a definir sus propias políticas y estrategias sustentables de producción, distribución y consumo de alimentos que garanticen el derecho a la alimentación para toda la población, con base en la pequeña y mediana producción, respetando sus propias culturas y la diversidad de los métodos campesinos, pesqueros e indígenas de producción agropecuaria, de comercialización y de gestión de los espacios rurales, en los cuales la mujer desempeña un papel fundamental" (Vía Campesina Internacional, 2008 citado en Centro de Estudios para la Democracia, 2016, p. 10).

En este análisis se plantea la tesis que los limitados espacios naturales en manos de las poblaciones indígenas, están mejor protegidos y conservados. Es decir, el respeto que los pueblos originarios practican con la naturaleza es superior a la práctica de sociedades industriales que saquean los bienes comunes, reducen los espacios públicos, eliminan las libertades ancestrales y socaban el conocimiento ancestral y la soberanía de las poblaciones rurales.

Desconocer la sabiduría atávica y vernácula es el principal riesgo al que se han sometido los procesos de activismo rural del desarrollo. La forma equívoca de transferir conocimiento y tecnificación agroalimentaria a las zonas rurales, deja en riesgo los sistemas alimentarios. Asimismo, los avances bioquímicos, bio-alimentarios y biogenéticos se han fundamentado en la dimensión económica, vulnerando la 
soberanía, y las libertades sociales. Maffi (2005) define que "la manifestación actual de la co-evolución son los elaborados sistemas de conocimientos, prácticas y simbologías que poseen las comunidades ancestrales. Los sistemas bioculturales forman parte indispensable de la memoria y del patrimonio de la humanidad y biodiversidad" (p. 605).

Los pueblos autóctonos han sido históricamente violentados en sus propios territorios, sometiéndoles así a diferentes procesos de a-culturización en los que la principal fuerza es la destrucción de sus culturas, cosmovisiones, tradiciones, costumbres y libertades sociales. Lo anterior ha sido denunciado en las campañas del Movimiento Agroecológico de América Latina y el Caribe (MAELA), celebradas desde 1998 en México, Argentina, Brasil, Bolivia, Chile, Colombia, Guatemala y Honduras.

En el abordaje de SA en procesos de desarrollo, es necesario revisar los marcos legales y políticos de aspectos relevantes como el manejo de recursos fitogenéticos, semillas criollas, defensa de territorios y protección del ambiente. Honduras cuenta con una política, ley y estrategia de seguridad y soberanía alimentaria y nutricional. Sin embargo, su implementación, seguimiento y evaluación ha sido limitada.

Entre 2014 y 2016, en Honduras 1.4 millones de personas padecían de hambre y el $22.7 \%$ de menores de 5 años presentaban retraso de crecimiento. Esta condición de inseguridad alimentaria es reflejo de la condición de pobreza (64.3\%) y pobreza extrema (40.7\%), acentuada en las zonas rurales (69.3\%) (Instituto Nacional de Estadística, INE, 2017).

El sistema agroalimentario hondureño se caracteriza por una producción de exportación (banano, café, camarones, melones, caña de azúcar y palma aceitera), y de alimentos básicos (maíz y frijol), provenientes de la agricultura familiar. En el patrón alimentario de los hondureños, cerca del $80 \%$ de las calorías provienen de solo 10 alimentos, siendo el maíz y los frijoles el $81 \%$ del consumo total de calorías diarias (Organización de las Naciones Unidas para la Alimentación y la Agricultura, 2016).

Aunque los marcos legales y políticos de Honduras definen la SA, su implementación es limitada, ya que el país no aplica las normativas para asegurar la toma de decisión acertada en términos de soberanía. Este comportamiento del Estado 
hondureño ha sido generalmente justificado por las emergencias de desastres naturales y crisis alimentarias de la población. Los datos estadísticos oficiales sobre soberanía alimentaria en Honduras son limitados, sin embargo, se estima que en la actualidad muy pocos alimentos de los producidos en el país son nativos y que de alguna manera han sido manipulados genéticamente. Por ejemplo, en el caso del cacao, del 100\% de lo producido de manera formal, el $66 \%$ es Trinitario y $34 \%$ es Forastero. Sobre la semilla criolla del cacao no se identifica ninguna producción formal, encontrándose únicamente en traspatio (huertos familiares) con no más de 20 árboles (Sánchez, 2015).

Los principales desafíos que enfrenta Honduras en términos de soberanía y seguridad alimentaria están vinculados a la explotación de minería metálica y no metálica que abarcan un $43 \%$ de la superficie boscosa (41,416 hectáreas) y un $34 \%$ de la extensión territorial agropecuaria (32,648 hectáreas). Sumando el 2\% de superficie agroforestal (1,935.60 hectáreas), se concluye que el 79\% de la superficie concesionada para la explotación minera metálica y no metálica se realiza en áreas agropecuarias, en bosques y zonas agroforestales, es decir, en 75,999.61 hectáreas que tienen traslape con límites locales percibidos como forestales y agropecuarios (Palma-Herrera, 2 018; 2019).

\section{Biodiversidad y desarrollo}

En la Política Nacional de Biodiversidad 2019-2029 (Mi Ambiente, 2019) se expresa que el Estado reconoce que la diversidad biológica es la clave para el desarrollo de nuestra población, porque suple las necesidades básicas a la sociedad, al permitir que sus servicios ecosistémicos sean fuentes de aprovisionamiento de alimentos, salud humana y aspectos culturales.

Honduras ocupa una posición geográfica privilegiada en la franja tropical del Neotrópico, el tener relación con dos océanos sus condiciones climáticas son ideales para generar biodiversidad, mientras que por estar entre Norte América y Sur América constituye el puente de paso entre la flora y fauna surgida en ambas regiones, permitiendo que ahí converjan esas especies. El mapa de ecosistemas enlista 63 para Honduras (House y Mejia, 2002), existiendo nueve sitios Ramsar y ocho humedales de importancia denominados por la North American Wetlands Conservation Council (NAWCC) (Mostacedo, 2008). También, son cuatro las Reservas de Biósfera incluidas en el Programa Hombre y Biósfera $(\mathrm{MaB})$ de la UNESCO. El Sistema Nacional de Áreas Protegidas de Honduras (SINAPH) cuenta con 91 Áreas Protegidas (AP), que 
ocupan una extensión aproximada de 3.9 millones de hectáreas, equivalentes al 36\% del territorio (Mi Ambiente, 2019).

Se estima que la población de Honduras es de 9.588 millones de habitantes (Instituto Nacional de Estadística, 2017) con una alta diversidad cultural reflejada en la coexistencia de nueve grupos étnicos: Garífunas, Lencas, Negros de habla inglesa, Misquitos, Tawahka, Pech, Chortis, Nahoas, Tolupanes, cuya población conjunta asciende a 600,000 habitantes aproximadamente, en su mayoría ubicados en territorios con una gran biodiversidad.

La Política Nacional de Biodiversidad 2019-2029 procura alinear sus objetivos con los Objetivos de Desarrollo Sostenible (ODS), intentando generar las pautas para establecer mejores estándares en el manejo y uso de los recursos naturales e impulsar medidas que ayuden a la restauración de los ecosistemas.

Con basta riqueza natural, el Estado se ha propuesto postular a Honduras, ante el Centro de Seguimiento de la Conservación Mundial (WCMC), perteneciente al Programa de Naciones Unidas sobre el Medio Ambiente (PNUMA), como país megadiverso y para ello, varios esfuerzos se han realizado tratando de alcanzar la información requerida. Sin embargo, el apoyo de recursos destinados por el país no es consistente y la asignación para la investigación sobre biodiversidad se limita a fondos de cooperación externa, por lo que el esfuerzo queda plasmado en un cúmulo de buenas intenciones.

La rica biodiversidad de Honduras ha sido afectada por diversos factores, particularmente el deterioro y destrucción de los hábitats naturales. Es por ello que el país forma parte del "hot-spot" o ecosistema crítico (EC), considerado una región con alta concentración de endemismo y de prioridad para la conservación de la biodiversidad (Mittermeier et al., 2004).

El deterioro sistemático de la biodiversidad en el país data de más de tres décadas, donde los gobiernos han dado poca atención a estos temas en su agenda. Es así, que más de veinte áreas solo están propuestas como áreas protegidas en el SINAPH. Más grave aún, el espacio más extenso continuo de bosque latifoliado en La Moskitia, que contiene las más grandes muestras de biodiversidad y que forma parte del Corredor Biológico Mesoamericano, se ha destruido en más del 60\%. Muchas 
tierras que fueron ocupadas por pueblos indígenas ya son grandes extensiones de potreros dispuestos a la ganadería o en condición de abandono. Como ya había sido denunciado por Suazo (2005), la apertura de carreteras en forma desmedida y sin autorización, ha permitido que como "efecto comején" la naturaleza sea devastada.

La dinámica de la biodiversidad no funciona por sí sola. La dimensión ambiental requiere ser fortalecida por la gobernanza, donde se llegue a arreglos institucionales que rijan el uso de los recursos naturales, ya sea por individuos o instituciones, con el fin de cumplir objetivos de sostenibilidad.

El tema ambiental en Honduras debe enfocarse de forma transdisciplinar, después de caracterizar las situaciones particulares para cada uno de los sitios y poblaciones que hacen uso de la biodiversidad. Es así que se debe de enfatizar en tres espacios que merecen atención: el humano, el ambiental y el legal:

a.Humano: La naturaleza provee al ser humano de alimentos, fármacos, agua y recursos indispensables para satisfacer sus necesidades básicas y, por lo tanto, para disfrutar de diversos derechos como la salud, la alimentación y la vivienda (Consejo Nacional de los Derechos Humanos, 2016). La importancia del vínculo entre biodiversidad y derechos humanos radica en que la pérdida de especies pone en riesgo la calidad de vida de las personas.

Somos la consecuencia receptora del Covid-19, siendo la causa de su origen nosotros mismos (Lagos-Witte, Portillo \& Suazo, 2020). En el documento Perspectivas del Medio Ambiente Mundial GEO-4, el Programa de Naciones Unidas para el Medio Ambiente (PNUMA, 2012) advierte que los cambios en la biodiversidad afectan a la salud humana. De acuerdo con Wilcox \& Ellis (2006), un número mayor de estudios demuestran la relación entre enfermedades emergentes infecciosas con las alteraciones producidas en la cubierta vegetal y en la utilización de la tierra como parcelación y urbanización. De acuerdo con estos autores, tres cuartas partes de las enfermedades infecciosas emergentes reconocidas fueron una vez o son actualmente, zoonóticas, entre ellas el Ébola, VIH, SAR, Fiebre Amarilla, Dengue y muchas otras. Las cosmovisiones que separan la naturaleza y la cultura son una causa subyacente de la pérdida de la diversidad biológica, ya que las culturas condicionan los comportamientos y enmarcan las relaciones de las personas con otras personas y con el mundo natural. Los valores bioculturales forman parte indispensable de la memoria y del patrimonio de la humanidad, así como de la diversidad de los territorios que habitan (Lagos-Witte et al., 2021). Los sistemas de valores holísticos y diversos, y 
las formas de vida de los pueblos indígenas y las comunidades locales, en todo el mundo ofrecen visiones culturalmente distintivas de futuros sostenibles alternativos que deben ser entendidos, respetados y protegidos a lo largo de todos los sistemas del gobierno, la economía y la sociedad (Peoples Forest, 2020).

Pero la importancia de la biodiversidad, radica en esta relación del campo con la ciudad, en las áreas naturales con las citadinas, en el bienestar mismo que genera la naturaleza en un contexto amplio a aquellos que no viven permanentemente en la naturaleza, por ejemplo, el agua generada y la estabilidad de los suelos y el clima. Por lo tanto, la biodiversidad es vital para cada hondureño, desde el que vive en La Moskitia hondureña, hasta el que ocupa un curul en el Congreso Nacional.

b.Ambiental: Puesto que la crisis ambiental se ha percibido como una fase acelerada, provocada por la humanidad (era del Antropoceno), cabe destacar que esta crisis ha existido desde siempre, pero no vista necesariamente como un tema crucial, sino como resultados acumulativos entre la interacción sociedad-naturaleza y los procesos biogeoquímicos propios de la misma naturaleza (Marin-Rivera, Murillo-Lopera, Rodríguez-Delgado, \& Martínez-Girón, 2018). Es así que la dimensión ambiental del desarrollo se refiere al conjunto de valores, actitudes y motivaciones que rigen las relaciones entre la sociedad y la naturaleza, y las formas como dichas relaciones se traducen en los sistemas de producción y apropiación de los recursos productivos, así como en el manejo individual y colectivo de los recursos naturales, el equilibrio ecológico y la preservación y calidad de la vida (Utria, 1986).

En Honduras, las políticas nacionales en el ámbito rural se han caracterizado por el limitado interés sobre la importancia del patrimonio natural del país como motor de desarrollo, muchas veces reduciéndose a ser visto como un espacio de extracción de recursos que apalancan de forma emergente los indicadores económicos. Los gobiernos se han centrado en la explotación de los recursos naturales que generan altos ingresos en el corto plazo, pero con un altísimo costo ambiental a mediano y largo plazo, tal como es el caso de la minería o la explotación forestal y la ganadería extensiva.

Espacios de gran importancia nacional e internacional para la conservación, son expuestos a la depredación y la explotación no sostenible, obedeciendo a intereses alejados de una visión sostenible. Para Dichdji (2017), los problemas ambientales persisten, dado que para revertir la situación se requiere de una transformación 
de los modos de producción y consumo, lo que implica reflexionar sobre nuestra organización y práctica social.

c.Legal: Según Vallejo (2014), lo legal se sitúa en la confrontación continua entre los procesos sociales que conllevan al uso de los recursos naturales que giran en torno a la compatibilidad y la coherencia de la conducta de la sociedad frente al cumplimiento del marco jurídico ambiental. El incumplimiento de este marco jurídico se debe, a que hasta ahora se ha considerado que se cuenta con suficiente disponibilidad real y potencial de los recursos que ofrece la naturaleza, los cuales no han sido regulados en tiempo y forma. Lo anterior, se vuelve una práctica habitual en un contexto jurídico emergente, disfuncional y selectivo, alejado de la finalidad de una política pública coherente con los objetivos de la sostenibilidad.

Honduras cuenta con más de 50 Tratados Internacionales en materia ambiental y más de 100 espacios jurídicos nacionales con el mismo fin. La Constitución de la República establece que los Tratados Internacionales celebrados por Honduras con otros Estados, una vez siendo ratificados por el Congreso Nacional, forman parte de su derecho interno. Debido a este alto rango jerárquico que ostentan, resulta necesario identificar aquellos que estén más estrechamente relacionados con el tema de biodiversidad.

La sociedad civil juega un papel importante en cuanto al combate de los ilícitos contra la biodiversidad, siempre que se capaciten y conozcan los mecanismos de denuncia. En este contexto la sociedad civil, los servidores públicos en temas ambientales, desde técnicos hasta jueces, deben desarrollar habilidades en los mecanismos de implementación de la ley.

\section{CONCLUSIONES}

Sin duda alguna, estamos ante el eminente reto de comprender el impacto del Antropoceno y del inicio de un cambio de paradigma, dado que la creciente economía basada en el consumismo sin límites no ha logrado satisfacer las necesidades de bienes y servicios de miles de millones de personas, aumentando de manera desmedida la desigualdad social y la pobreza. Todo parece indicar que mientras se mantenga este patrón de comportamiento humano, acontecimientos como el cambio climático y el calentamiento global tendrán mayor impacto en todos los campos de la vida en la tierra. Como se ha expuesto en el análisis a lo largo de esta publicación, para que 
pueda existir un verdadero desarrollo humano sostenible se tendrán que afrontar grandes desafíos actuales y futuros, entre los que se encuentran los siguientes: cambios en la conciencia ambiental, nuevos valores éticos de producción y consumo, nueva dimensión de la educación ambiental, revalorización de las culturas ancestrales y revitalización de sus lenguas y tradiciones, así como la protección de los diferentes ecosistemas, las personas y comunidades que se dedican a protegerlos. Por lo tanto, es insoslayable encontrar la convergencia entre los pilares económicos, sociales, ambientales y políticos.

El relacionamiento teórico entre soberanía alimentaria, ambiente y desarrollo es un ejercicio al que varias escuelas de pensamiento han aportado y aun cuando estas posiciones epistemológicas se interrelacionan, el debate se mantiene en disyuntiva y con significados inefables. Es así que estos conceptos convergen en espectros relacionados al manejo y aprovechamiento de los bienes naturales, la defensa del territorio y el ambiente, la soberanía alimentaria y los sistemas alimentarios rurales, entre otros. Por lo tanto, darles voz y rostro a los pueblos indígenas y rurales debe ser prioridad en las construcciones teóricas que replanteen el abordaje temático orientado al rescate, protección y preservación de la cultura y la soberanía alimentaria, impulsando el desarrollo humano sostenible.

La agricultura, por un lado, ha permitido satisfacer la demanda de alimentos de las sociedades, pero a la vez, ya sea por baja tecnificación o por su intensificación es contributiva a la deforestación, contaminación ambiental y generación de gases de efecto invernadero. Ante este dilema, la agricultura debe asegurar la producción de alimentos para una población cada vez mayor, pero a la vez adaptarse a un clima cada vez más variable.

Honduras se encuentra en un espacio geográfico rico en diversidad biológica y cultural, el cual requiere de manera urgente que las políticas públicas generadas en diferentes dimensiones del desarrollo y/o de crecimiento económico, puedan ser implementadas de manera eficiente y de forma coherentes con los Objetivos del Desarrollo Humano Sostenible. Es imperativo hacer cambios integrales y profundos en los procesos democráticos del país que mejoren la transparencia y que castiguen la corrupción dentro de un "Estado fallido". Por todo lo anteriormente expuesto debe verse a la biodiversidad como una oportunidad única de lograr espacios de equidad social y justicia ambiental, ya que estos recursos son la fuente renovable que podría generar ingresos sostenibles a gran parte de la población y contribuir al logro del bienestar de los hondureños que hoy viven en condiciones de alta vulnerabilidad ambiental y social. 


\section{AGRADECIMIENTO}

Los autores desean expresar su agradecimiento a la Dra. Olga Lucia Sanabria, al Dr. Alejandro Casas y a la Dra. Sonia Lagos-Witte por compartir sus investigaciones científicas y sus experiencias de campo que han enriquecido el debate científico sobre el tema de esta publicación. La supervisión académica estuvo a cargo de la Dra. Sonia Lagos-Witte, a quien agradecemos su dedicación y apoyo a nuestro trabajo.

\section{BIBLIOGRAFÍA}

Aznar, P., \& Barrón, Á. (2017). El Desarrollo Humano Sostenible: un compromiso educativo. Ediciones Universidad de Salamanca, 25-53.

Banco Central de Honduras. (2020). Banco central de Honduras. Obtenido de htpps:/ www.bch.hn

Banco Interamericano de Desarrollo. (2019). Analisi de políticas agropecuarias en Honduras. Tegucigalpa, Honduras: BID. doi:http://dx.doi.org/10.18235/0002274 Banco Mundial. (s.f.). Agricultura inteligente con respecto al clima. Recuperado el 19 de junio de 2021, de BM: https://www.bancomundial.org/es/topic/climate-smartagriculture

Centro de Estudios para la Democracia. (2016). Seguridad y soberania alimentaria: ¿Que hay detras de los tratados internacioanlaes y políticas públicas en Honduras? Tegucigalpa: CESPAD.

CNN. (9 de Junio de 2021). Perspectivas desde Mexico. Obtenido de Archivo de video: https://www.youtube.com/watch?v=ekmeVae12vg

Consejo Nacional de los Derechos Humanos. (2016). Biodiversidad y derechos humanos. Mexico: Trigeminum.

Deutsche Welle. (7 de julio de 2020). Nuevo récord de deforestación en Amazonía brasileña en primer semestre de 2020. Recuperado el 19 de junio de 2021, de Deutsche Welle: https://www.dw.com/es/nuevo-r\%C3\%A9cord-de-deforestaci\%C3\%B3n-enamazon\%C3\%ADa-brasile\%C3\%B1a-en-primer-semestre-de-2020/a-54133765

Dichdji, A. (2017). Naturaleza y cultura: Dialogos interdisiplinarios entre la historia ambiental y la antropologia. Luna Azul(44), 277-293. doi: 10.17151/luaz.2017.44.17 
Gómez, T. (29 de Julio de 2020). Mongabay: periodismo ambiental independiente en Latinoamérica. Obtenido de Latinoamérica: región donde más defensores ambientales fueron asesinados en 2019: https://es.mongabay.com/2020/07/latinoamerica-masdefensores-ambientales-asesinados-global-witness-2019-informe/

Guhl,A.(2012). Biodiversidad, Conservaciony Desarrollo.BIodiversidady Desarrollo, 379-408. Obtenido de http://www.jstor.org/stable/10.7440/j.ctt19qgdmr.17

Guzmán, E. S. (2008). Agroecología y agricultura ecológica: hacia una "re" construcción de la soberanía alimnetaria. (U. d. Rabanales, Ed.) Instituto de Sociologia y Estudios Campesinos, 12. Obtenido de https://core.ac.uk/download/pdf/60859835. pdf

Hodge, C., Daber, M., López, R., Castilla, J., \& Edwards, G. (2018). Desarrrllo humano integral y sostenible. (P. U. Chile, Ed.) Teología y vida(59), 399-430.

Honduras., B. C. (2018). Programa Monetario 2016-2017. Banco Central de Honduras $(\mathrm{BCH})$. Tegucigalpa, Honduras: Banco Central de Honduras (BCH).

House, P., \& Mejia . (2002). Mapa de ecosistemas vegetales para Honduras. AFE/ COHDEFOR. Tegucigalpa: Proyecto PAAR.

Instituto Nacional de Estadística. INE. (2017). Encuesta Permanente de Hogares de Propósitos. Tegucigalpa, Honduras.: Instituto Nacional de Estadística. Obtenido dehttps:/www.ine.gob.hn/images/Productos\%20ine/TrifoliosHogares/2018/ Boletin\%20Pobreza\%202018.pdf

Iranzo, J. M. (2005). Limits to grow: 30 years of day. Empiria-Revista de metodología en ciencias sociales(10), 227-245.

Lagos Witte, S., Portillo, H. O., Suazo Euceda, J. P., \& Suazo, A. M. (23 de Junio de 2020). Pandemia Vs Biodiversidad: Un resultado incierto. Obtenido de https://www. youtube.com/watch?v=i0geOO9a4RM

Lagos-Witte, S., Sanabria, O. L., \& Casas Fernández, A. (13 de mayo 2021). Retos actuales para la conservacion de la biodiversidad en Honduras y sus efectos en la gestión del desarrollo humano sostenible. Debate contemporaneo sobre las Ciencias de Desarrollo Humano. Tegucigalpa. Obtenido de https://www.youtube. $\mathrm{com} /$ watch? $\mathrm{v}=\mathrm{u} 0 \mathrm{MeOuYmr} 4 \mathrm{Y} \&$ list $=\mathrm{PLazH} 7 \mathrm{~s} \_O T o Q B I Y 4 F H 477 \mathrm{Mysrqj}-$ 7wMT4Q\&index $=4 \& a b \_$channel=OBSANUNAHOBSANUNAH 


\section{DESAFÍOS CONTEMPORÁNEOS DEL DESARROLLO HUMANO Y SOSTENIBILIDAD: UNA MIRADA DESDE LA BIODIVERSIDAD, AGRICULTURA Y SOBERANÍA ALIMENTARIA.}

Linares-Rosas, M., Goméz, B., Adalsoro-Maya, E., \& Casas, A. (2021). Nahua biocultural richness: an ethnoherpetological perspective. Journal of Ethnobiology and Ethnomedicine(33). doi:https://doi.org/10.1186/s13002-021-00460-1

Maffi, L. (2005). Linguistic, Cultural, and Biological Diversity. Terralingua, Salt Spring Island, British Columbia V8K 2N6, Canada: The Annual Review of Anthropology.

Marin-Rivera, J., Murillo-Lopera, K., Rodriguez-Delgado, I., \& Martínez-Girón, J. (2018). La agroecologia: Alternativa de desarrollo sustetable ante la crisis ambiental en un mundoglobalizado. Revista de investigación Agraria y Ambiental, 9(2), 63-76. doi:http://dx.doi.org/10.22490/21456453.2196

Mi Ambiente. (2019). Politica Nacional de Biodiversidad 2019-2020. Tegucigalpa: Mi ambiente.

Mitteermeier, R., Robles, G., Hoffman, M., Pilgrim, J., Brooks, T., Mittermeier, c., . . . Da Fonseca, G. (2004). Hotspots Revisited. Mexico City: CEMEX.

Morazán, P. (2017). Honduras frente a los retos de la agenda 2030 para el Desarrollo Sostenible. Perspectivas del Desarrollo, 9-22. Obtenido de https://www.camjol.info/ index.php/RPDD/article/view/9379

Mostacedo, S. (2008). Caracterizacion del estado actual de los humedales de importancia internacional de Honduras. Escuela Agricola Panamericana : Zamorano .

Nájar, A. (2016 de octubre de 2016). Los daños ocultos que provoca el aguacate, el "oro verde de Mexico". Recuperado el 19 de junio de 2021, de BBC: https:/www. bbc.com/mundo/noticias-america-latina-37581668

Olazával,H.(2014). Los orígenes de la agricultura: nuevos paradigmas. Investigaciones sociales, 18(33), 53-86.

Organizacion de las Naciones Unidas. (2018). Acuerdo de Escazú. Acuerdo regional sobre el acceso a la información, la participación pública y el acceso a la justicia en asuntos ambientales en América Latina y el Caribe. New York, USA: Organizacion de Naciones Unidas.

Organización de las Naciones Unidas para la Alimentación y la Agricultura. (2013). Ley marco derecho a la alimentación, seguridad y sobernía alimentaria. Panamá: FAO. Obtenido de http://www.fao.org/fileadmin/templates/righttofood/documents/ 
project_m/doc/Ley_Marco_DA_Parlartino.pdf

Organización de las Naciones Unidas para la Alimentación y la Agricultura. (2016). Informe Estado del Hambre FAO, 2015. Tegucigalpa, Honduras.: FAO.

Palma-Herrera, J. L. (2018). Impulsando la paz y la protección del ambiente en el país más peligroso del mundo: estudio geográfico para evitar el impacto negativo de la minería en Honduras. Geografías al servicio de los procesos de paz. La Paz, Bolivia.: ResearchGate.

Palma-Herrera, J. L. (2019). El regreso de los enclaves a Honduras en el siglo XXI: Las zonas de empleo y desarrollo económico (ZEDE), la versión de las ciudades Charter rechazadas por su creador Paul Romer (2010-2019). San José, Costa Rica.: Anuario de Estudios Centroamericanos, Universidad de Costa Rica.

Peoples Forest. (2020). Las Perspectivas Locales sobre la Diversidad Biológica 2 - Las contribuciones de los pueblos indígenas y las comunidades locales a la implementación del Plan Estratégico para la Diversidad Biológica 2011-2020 y a la. Reino Unido: Peoples, Forest. Obtenido de https://www.cbd.int/gbo/gbo5/ publication/lbo-2-summary-es.pdf

Perfecto, I., \& Vandermeer, J. (2008). Biodiversity Conservation in Tropical Agroecosystems, a new conservation paradigm. (U. o. School of natural resources and environment, Ed.) Annals of the New York Academy of Sciences, 173-200. Obtenido de https://www.researchgate.net/publication/5290721_Biodiversity_Conservation in_Tropical_Agroecosystems

PNUD. (2020). Informe sobre desarrollo humano 2020. La próxima frontera:Desarrollo humano y el antropoceno. Tegucigalpa, Honduras: Programa de las Naciones Unidas para el Desarrollo.

PNUMA. (2012). Perspectivas del medio ambiente Mundial, Medio ambiente para el futuro que Queremos. Panama: PNUMA.

Rendón, J. (2007). El desarrollo humano sostenible: ¿un concepto para las transformaciones? Equidad y desarrollo,. Equidad y desarrollo(7), 111-129. Obtenido de https://www.redalyc.org/pdf/957/95700707.pdf

Rodrigo, A. (2015). El Desafio del Desarrollo Sostenible: los principios del Derechon internacional relativos al desarrollo sostenible. Madrid, España: Ediciones Jurídicas y Sociales. Obtenido de https://www.marcialpons.es/media/pdf/9788416402311.pdf 
Sánchez, J. H. (2015). Informe Final del proyecto garantizando una alta calidad del cacao orgánico a lo largo de la cadena de valor en Honduras. . La Lima, Cortés, Honduras: Fundación Hondureña de Investigación Agrícola, FHIA.

Serrano, A., \& Stein, A. (2019). Re-construyendo la ciudad: El espacio público como un lugar de simetría, diálogo y trscendencia. Honduras: UNAH.

Suazo, J. P. (2005). Percepcion y uso de la vida silvestre: caso de ladinos y Tawahkas en el Corredor Biologico Mesoamericano. Tegucigalpa: Guaymuras.

Utria, R. (1986). La dimension ambiental del desarrollo y su planificacion. Bogota: Linotipia. Obtenido de https://repositorio.cepal.org/bitstream/handle/11362/28257/ S30131\%20U92_es.pdf

Vallejo Larios, M. (2014). Problematica ambiental en Honduras: respuestas desde el derecho. Revista de Derecho, 35(1), 27-49. Obtenido de 1782-Texto del artículo-6384-1-10-20150130 (1).pdf

Vila, R. (2015). La era del desarrollo sostenible. Barcelona, España: Grupo Planeta. Obtenido de https://www.planetadelibros.com/libros_contenido_extra/31/30978_ La_era_del_desarrollo_sostenible.pdf

Wilcox, B., \& Ellis B. (2006). Forests and emerging infectious diseases of humans. (FAO, Ed.) Unasylva, 57, 11-18.

WWF. (2020). Informe Planeta Vivo 2020: revertir la curva de la pérdida de biodiversidad. Resumen. (R. Almond, R. Grooten, \& T. Petersen, Edits.) Gland, Suiza: WWF. Obtenido de https://wwfes.awsassets.panda.org/downloads/ infomeplanetavivo_2020_resumen_1.pdf.

\section{CÍRCULO TEMÁTICO: AMBIENTE Y DESARROLLO}

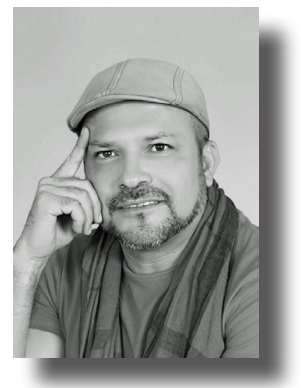

Juan Pablo Suazo

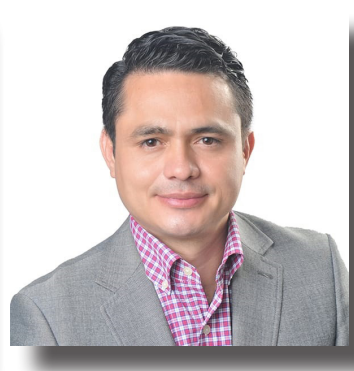

Marvin Moreno

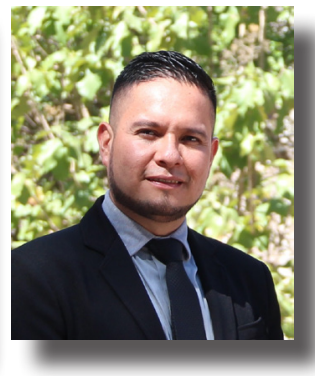

José Daniel Lara

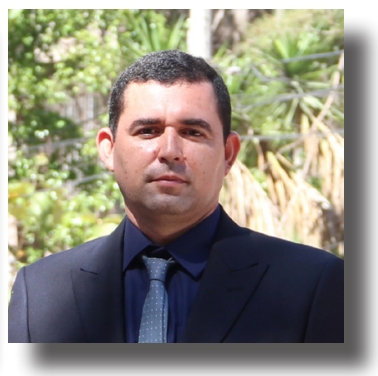

Pablo Díaz 